\title{
La alfabetización digital en el público adulto mayor. Un acercamiento desde la comunicación de las relaciones públicas en Perú.
}

The senior citizen public digital literacy. An approach from public relations communication in Peru

\author{
Alfabetização digital no público idoso. Uma \\ abordagem desde a comunicação de relações públicas \\ no Peru
}

\author{
Luz María Flores Cabello ${ }^{1}$ \\ Universidad de San Martín de Porres \\ lfloresc@usmp.pe
}

Fecha de recepción: 6 de agosto de 2020

Fecha de recepción evaluador: 12 de agosto de 2020

Fecha de recepción corrección: 16 de agosto de 2020

\begin{abstract}
Resumen
Dando inicio a la tercera década del presente milenio y dejando atrás una segunda década en la que la internet y las nuevas tecnologías de la información y la comunicación (TIC) nos abrieron innumerables puertas de acceso al conocimiento y la conexión, surge la

\footnotetext{
${ }^{1}$ Luz María Flores es profesora e investigadora en la Facultad de Ciencias de la Comunicación de la Universidad de San Martín de Porres y doctoranda en el programa de Periodismo de la Universidad Complutense de Madrid. ORCID: https://orcid.org/0000-0003-4272-6419
} 
interrogante de si la expansión de esta tecnología dará paso a la inclusión de aquellos que todavía no se han podido insertar en esta sociedad de la información y el conocimiento.

La realidad muestra que el mundo cada día se hace más viejo de forma más rápida y en casi todas partes, por lo que es evidente que las personas adultas mayores son las llamadas abrir y explorar esta nueva etapa de la vida humana a partir del uso y consumo de las TIC.

El presente artículo ofrece una mirada del estado actual de la alfabetización digital en el colectivo adulto mayor de Perú a través del análisis de contenido de sus políticas públicas orientadas a la alfabetización digital de los adultos mayores, con las que se deben desarrollar los procesos comunicativos entre las organizaciones y el público adulto mayor. En el mismo sentido, se revisa la documentación existente en Perú sobre el marco del acceso a las tecnologías de la información y la comunicación que promueven las instituciones públicas para una adecuada alfabetización digital del público adulto mayor.

De este modo, se presenta una tabla de análisis de contenido de los recursos hallados en la investigación, y se constata una falta de visibilidad del tema, dejando como evidencia que la tan repetida frase de la inclusión social, no se aplica desde la perspectiva de la tecnología de la información y la comunicación para las personas adultas mayores.

Palabras clave: alfabetización digital; sociedad de la información; comunicación interactiva; relaciones públicas.

\begin{abstract}
Starting the third decade of this millennium and putting a second decade which internet and information new technologies and communication (ICT) on one side, they opened countless gates to reach to knowledge and connection, an unanswered question emerges whether this technology expansion lay the foundations to include those who could not be inserted in this information and knowledge society yet.
\end{abstract}

Reality shows that the world gets older every day at a more rapid rate, everywhere. Due to that situation it is evident that senior citizens are called to open and explore this new human life stage from the ITC use and consumption.

The current article takes a look of the contemporary digital literacy situation of the Peruvian senior citizens collective through the public politics analysis content, oriented to senior citizen digital literacy, with which it must develop the communicative process between the organizations and the senior citizens. In the same way, the revision about the existing documentation in Peru related to information technologies and communications access framework that public institutions promote in order to have a suitable senior citizen digital literacy.

Thereby, a table of analysis content of found resources in the research, and a lack of exposure of the topic is presented, leaving as evidence the phrase repeated endlessly about social inclusion which is no applied from information and communication technology perspective for senior citizens. 
Key words: digital literacy, information society, interactive communication, public relations.

\section{Resumo}

Começando a terceira década do presente milênio e deixando para trás uma segunda década em que a Internet e as novas tecnologias da informação e comunicação (TIC) abriram inúmeras portas de acesso ao conhecimento e à conexão, aparece/ surge a questão de saber se a expansão dessa tecnologia levará à inclusão daqueles que ainda não foram capazes de entrar nessa sociedade da informação e do conhecimento.

A realidade mostra que o mundo está envelhecendo mais rápido e em quase todos os lugares, tornando evidente que os idosos são chamados a abrir e explorar esse novo estágio da vida humana a partir do uso e consumo das TIC.

Este artigo oferece uma visão do estado atual da alfabetização digital em idosos no Peru por meio da análise de conteúdo de suas políticas públicas, dirigidas a alfabetização digital de idosos, com as quais os processos de comunicação entre organizações e o público idoso devem ser desenvolvidos.

No mesmo sentido, é revisada a documentação existente no Peru sobre o marco de acesso às tecnologias da informação e comunicação promovida pelas instituições públicas para uma adequada alfabetização digital do público idoso.

Dessa forma, é apresentada uma tabela de análise de conteúdo dos recursos encontrados na pesquisa e podese verificar a falta de visibilidade do tópico, deixando como evidência que a frase tão repetida de inclusão social não é aplicada na perspectiva de tecnologia da informação e comunicação para idosos.

Palavras chave: alfabetização digital; sociedade da informacao; comunicacao; interativo; relacoes públicas.

\section{Introducción}

Sumergidos en la dinámica de una sociedad red como la denomina Manuel Castells, donde los distintos nodos que la conforman son los grupos de individuos que las integran y complementan, de acuerdo a sus necesidades de relación que establecen unos y otros, surge la necesidad de identificar, conocer, entender y analizar cómo funciona la comunicación digital en los procesos comunicativos que se originan entre los individuos y sus nodos, a su vez, audiencias, públicos, colectivos, consumidores, etc.

Las tecnologías de la información y comunicación (TIC), son las grandes autopistas que dinamizan la comunicación en esta gran sociedad red en la que todos los actores que la conforman tienen algo que decir e intercambiar en una dinámica de interacción inmediata, personalizada y constante que, a su vez, busca la legitimidad de su saber hacer. Elementos como ordenadores, dispositivos personales multimedia, telefonía móvil, redes sociales, Internet, televisión digital, sistemas de navegación digital, ebooks 
entre otros, son las herramientas que conforman el sistema de las TIC, que como señala (Abad, 2017), son la suma de tres tecnologías que ya existían: las audiovisuales, las telecomunicaciones y las informáticas que unidas configuran la cultura digital que vivimos actualmente.

Según Juan Miguel Aguado, la experiencia de la comunicación mediada por la tecnología recompone la forma de mirar el mundo tanto por el emisor y receptor, planteándose nuevas estructuras de la experiencia asociadas al sentido en el mundo social. "Si rememoramos las fuentes de nuestra experiencia individual descubriremos que en grado y extensión la mayor parte de ellas proviene de dispositivos tecnológicos de mediación de la experiencia” explica. (Aguado, 2004) (p. 151)

La presencia e influencia de las nuevas tecnologías de la información que constituyen la sociedad de la información, están generando un impacto irreversible en los procesos comunicativos de los individuos creando nuevos entornos transmediáticos en los que esos grupos de individuos (nodos) se retroalimentan en mayor o menor medida del conocimiento de estas, lo que origina un desbalance en el acceso de la información y el conocimiento entre unos grupos y otros. En ese sentido, las tecnologías de la comunicación operan desde un nivel epistémico, en el que el sujeto se relaciona con el entorno y el nivel simbólico en el que la experiencia transforma y traspone los sentidos, a lo que (Aguado, 2004) denomina la meta-experiencia, es decir, una experiencia sobre experiencias, una manera simbólica de vivir otras experiencias de otros o 'experiencias vicarias', como las define el autor.

Insertados en pleno desarrollo de la tercera revolución industrial, donde prima la velocidad de la información, la interconexión, el movimiento, la globalización de la economía y los mercados, (Álvarez, 2013) el avance de la transformación digital constituye un reto continuo para todas las organizaciones. En la que todos (individuo, consumidor, fans, influencer) nos encontramos interconectados gracias a la Internet, y surgen nuevas formas de hacer comunicación hacia diferentes públicos con características muy distintas, como es el caso del público adulto mayor.

En esta sociedad red digital, conformada por miles de nodos entretejidos donde unos retroalimentan a otros y otros se unen y fragmentan continuamente, hay un público de individuos que el nuevo entorno tecnológico no ha facilitado su plena inclusión. Hoy en día, el grupo etario denominado los Baby boomer, aquellos nacidos después de la segunda guerra mundial hasta mediados de la década de los años 60 del siglo XX en Perú, son vistos como inmigrantes o analfabetos digitales. Personas que enfrentan los nuevos desafíos del aprendizaje tecnológico en la tercera edad de sus vidas, pero que, sin embargo, a muchos de ellos les permite desarrollar sus habilidades al máximo rendimiento.

La tarea por integrar a este público, como usuario y como generador de sus propios contenidos capaces de construir sus propios significados digitales, es una forma en la que la alfabetización digital cumple un rol imprescindible en esta gran sociedad de la información. El objetivo de la presente investigación es analizar y revisar las 
principales políticas públicas orientadas a los derechos de los adultos mayores en Perú desde la perspectiva de la comunicación pública y revisar la literatura existente en Perú sobre el marco de las tecnologías de la información y la comunicación en el que se vean expresados sus derechos a acceder a una alfabetización digital integral. Lo cual puede ser útil para orientar nuevas investigaciones y programas en entornos tecnológicos para los adultos mayores, dado que la producción intelectual desde la perspectiva comunicacional relacional sobre el adulto mayor es casi nula en Perú.

El trabajo está dividido en tres secciones. La siguiente sección describe las características de la sociedad de la información y el conocimiento usando fuentes de información supranacionales y nacionales; el concepto de la alfabetización digital de acuerdo con aportes de varios autores; y el público adulto mayor basado en fuentes de información supranacionales y nacionales. En la siguiente sección se desarrolla una tabla de análisis de contenido de los recursos hallados en la investigación correspondientes al marco normativo de Perú. En la sección final se presentan las conclusiones de la investigación.

\section{Marco Teórico}

\section{Sociedad de la Información}

La declaración de principios para construir una Sociedad de la Información para el nuevo milenio firmada en Ginebra, en el marco de la Cumbre Mundial de la Sociedad de la Información convocada por la Unión Internacional de Telecomunicaciones (UIT) de las Naciones Unidas en el 2003, da a conocer una serie de lineamientos y principios enfocados en las capacidades de las tecnologías de la información y la comunicación y su impacto en el desarrollo del individuo y de la sociedad en su conjunto. El documento enfatiza, por ejemplo, el compromiso de los países participantes de "construir una sociedad de la Información centrada en la persona, integradora y orientada al desarrollo en que todos puedan crear, consultar, utilizar y compartir la información y el conocimiento (...) en busca de su desarrollo sostenible y calidad de vida". (UIT, 2005). Poniendo en relieve la gran importancia de desarrollar mecanismos idóneos que involucren a millones de personas en todo el mundo indistintamente de su edad, condición o ubicación.

La Declaración de Ginebra se reafirma en que el fundamento esencial de la Sociedad de la Información es la libertad de opinión y expresión de todo individuo en el mundo, por lo tanto tiene derecho a investigar, recibir opiniones e informaciones y poder difundirlas por cualquier medio de expresión sin ser molestado porque, "la comunicación es un proceso social fundamental, una necesidad humana básica y el fundamento de toda organización social", y constituye el eje central de la Sociedad de la Información.

En esa línea hace hincapié en las capacidades que ofrecen las TIC para abrir oportunidades de desarrollo a los individuos con evidentes mejoras en su calidad de vida como, por ejemplo, las oportunidades laborales, la educación a distancia, el aprendizaje continuo a lo largo de la vida. En ese sentido, hace evidente la importancia de una 
alfabetización electrónica para promover las aptitudes en el ámbito de las tecnologías de la información y la comunicación tanto para personas con discapacidad, grupos desfavorecidos y vulnerables como para los individuos en general. Poniendo en relieve la importancia y uso masivo de la Internet por parte de los gobernantes que apunten al proceso de la construcción de una Sociedad de la Información.

Son varios los documentos y pasos previos que se fueron dando en el concierto internacional a la cabeza de las Naciones Unidas para dar forma al desafío global que significa la entrada al nuevo milenio con una sociedad informada y cada día más interconectada. Es así como, a la Declaración de los principios de Ginebra en el 2003, se sumó el Compromiso de Túnez firmado en 2005 en lo que conformó la segunda fase de la Cumbre Mundial sobre la Sociedad de la Información de las Naciones Unidas. En ella, se reafirma la importancia del uso de las TIC en todos los ámbitos de desarrollo con el consecuente compromiso para promover su acceso universal, ubicuo, equitativo y asequible con especial énfasis en la inclusión de los grupos vulnerables de la sociedad, como son "los emigrantes e inmigrantes, los desplazados internos, los refugiados, los desempleados, las personas desfavorecidas, los pueblos nómadas, las personas mayores y las personas con discapacidad". (UIT, 2005. Pg. 63).

En Perú, la primera iniciativa para crear una Comisión Multisectorial para el Desarrollo de la Sociedad de la Información (CODESI) surge en 2003 cuando se acuerda elaborar el primer Plan de Desarrollo de la Sociedad de la Información que luego de varias modificaciones terminó siendo publicado ocho años después en julio del 2011. Para ese entonces el documento final se titulaba Plan de Desarrollo de la Sociedad de la Información en Perú - La Agenda Digital 2.0 que contiene seis capítulos, glosario y anexos y que tiene como alcance de aplicación a todas las entidades integrantes del Sistema Nacional de Informática.

El documento centra la importancia de las TIC como el eje dinamizador para el desarrollo de la Sociedad de la Información y el Conocimiento en Perú no como un fin, sino como una herramienta transversal para el logro de los objetivos estratégicos planteados en el documento. En su marco estratégico rescata como su visión lo siguiente: "la sociedad de la información se ha transformado en una sociedad de la información y conocimiento, activa y productiva. Esta sociedad es integrada democrática, abierta, inclusiva y brinda igualdad de oportunidades para todos" (PCM, 2011). Y reconoce a las TIC como herramientas imprescindibles para el desarrollo social, tanto para un Estado moderno, transparente, participativo y ético, como para el desarrollo de la competitividad de las empresas y organizaciones.

Lo que bien señala (Castells, 2009) en su libro Comunicación y Poder, "una sociedad red es aquella cuya estructura social está compuesta de redes activadas por tecnologías digitales de la comunicación y la información basadas en la microelectrónica". (p.50) Explica una estructura social basada en la articulación de las TIC a nivel global, en la que no necesariamente participativas para todas las personas, dado que no toda la sociedad esta insertada en esa dinámica digital global, aunque sí se 
vea afectada por esta. Lo que significa que existe un alto sentido de exclusión originado entre otras razones por la falta de una efectiva red tecnológica inclusiva.

\section{Alfabetización Digital}

Lo que, para el común de las personas, alfabetización significa la capacidad de un individuo de aprender el alfabeto, las palabras, los números hasta saber leer y escribir desde sus primeros años de vida, hoy ese término ha ampliado su significado hasta llegar a las fronteras de las tecnologías de la información y comunicación, transformándose en alfabetización digital. En ese sentido, el concepto alfabetización se abre para entenderlo como la adquisición y dominio de competencias que permiten el uso de los símbolos de representación de la cultura en sus múltiples formas y lenguajes (Fundación Telefónica, 2012). Es decir, leer y escribir multimedia, la convergencia de la imagen, el sonido y el texto en un documento interactivo. (Gutierrez., 2003). Lo que viene a ser la capacidad de aprender a usar las herramientas tecnológicas desde un computador o un dispositivo móvil entre otros. Esto es, la nueva capacidad de volver a adquirir conocimientos de las nuevas tecnologías para aplicarlas en la vida diaria de las personas, en este caso, de las personas adultas mayores quienes se educaron en la formación tradicional.

Según el Plan de Desarrollo de la Sociedad de la Información en Perú - La Agenda Digital 2.0, este mismo término es entendido como 'alfabetización informacional'. Se pone en contexto que la alfabetización informacional requiere que el usuario cuente con las competencias necesarias para usar las TIC a fin de tener acceso a la información y poder crear otra, que puede ser de dos tipos, la alfabetización informática por el conocimiento de las técnicas de comunicación y la alfabetización en los medios de comunicación, por la comprensión de los diversos canales, medios y formatos por los que se transmite la información. (PCM, 2011, P.71)

En referencia a los principios citados en la Cumbre Mundial sobre la Sociedad de la Información de Ginebra, el término alfabetización digital se plantea como 'alfabetización electrónica' y 'alfabetización en el ámbito de las TIC'.

Paz (2008) afirma: "Se asume que se espera que el aprendiz adquiera competencias básicas en el uso del computador, especialmente en el uso de opciones generales del sistema operativo, así como programas básicos y el uso de los servicios de Internet" (p. 4). Cuando un individuo tiene la familiaridad de utilizar un correo electrónico o navegar por la web, ya se podría considerar una persona apta para el uso de las herramientas digitales

Considerando que el impacto de las TIC se registra en todos los niveles del desarrollo de las sociedades, todos los grupos de personas que conforman esa gran sociedad red, constituida por nodos más dinámicos unos que otros, necesitan ser alfabetizadas, incluidas e insertadas en esta nueva forma de adquirir conocimientos que ofrece la tecnología. (Area, 2015) (Travieso, 2008). Salta la reflexión de si el escenario de la alfabetización digital es horizontal y llega a todo tipo de individuos. Paz (2008) sostiene que hay dos destinatarios para la alfabetización digital, primero la niñez y todo 
el sistema educativo a su alcance y segundo, los adultos quienes están en la necesidad de un aprendizaje permanente y entre estos, los adultos mayores quienes no deben quedar relegados fuera de la sociedad de la información.

Es importante resaltar las dificultades que presenta la alfabetización digital, no únicamente desde una perspectiva tecnológica donde las condiciones técnicas se imponen, como es el caso de la accesibilidad de la Internet en lugares alejados de las grandes ciudades, sino desde la mirada sociocultural con respecto a la formación de ciudadanía en el contexto de la sociedad de la información (Fundación Telefónica, 2012), dado que la educación es la mayor responsable de la construcción democrática de las libertades de los individuos.

Según Gutiérrez (2003), entre los beneficios que trae la alfabetización digital en los adultos se puede destacar el sentido de mayor autoestima y satisfacción personal, mayor ejercicio del cerebro, de la memoria y el refuerzo de los conocimientos adquiridos.

Desde la creación del internet, las sociedades se encuentran en continuo cambio y movimiento, la sociedad red que describe Manuel Castells es cada vez más dinámica, veloz, fluctuante, hiperconectada y hasta cierto punto caótica, pero globalizada. La innovación tecnológica, ya no es una proyección a futuro, es una realidad, el desarrollo de las Tecnologías de la Información y la Comunicación (TIC) generan cambios profundos en la forma de trabajar, de estudiar y aprender de las personas. Cada día se crean nuevas empresas tecnológicas gracias al emprendimiento de profesionales de todas las disciplinas que buscan aplicar las TIC de manera novedosa a sus propuestas de negocio. La cultura de lo digital es una realidad ya insertada en la vida de todas las sociedades del mundo, aunque existen bolsones de individuos a los que aún no se les ha permitido insertarse plenamente a esta expansión.

\section{El Público Adulto Mayor}

La segunda Asamblea Mundial sobre el Envejecimiento, celebrada en 2002 por las Naciones Unidas en Madrid, adopta un plan de acción internacional sobre el envejecimiento para afrontar los retos de entrada al nuevo milenio. El documento establece tres directrices de acción a saber: las personas de edad y el desarrollo, la promoción de la salud y el bienestar de la vejez, y el logro de entornos emancipadores y propicios. (ONU, 2002)

El documento enfatiza que las sociedades están afrontando la transformación del cambio demográfico de manera mucho más rápida propiciando nuevos planteamientos que ponen en relevancia el valor del envejecimiento y la calidad de vida para el adulto mayor. En su artículo numeral 10, destaca el potencial de las personas adultas mayores como base para el desarrollo futuro y el aporte de su sabiduría que no solo le permite asumir la iniciativa de su propia mejora sino también, le permite participar activamente en toda la sociedad. De igual manera, destaca la participación de la población adulta mayor en la vida económica, política, social y cultural de la sociedad, teniendo la oportunidad de trabajar 'hasta que quieran y sean capaces de hacerlo' así como seguir 
teniendo acceso a la educación y los programas de capacitación. (p.9). es decir, la participación de las personas mayores en el concierto de la sociedad constituye un elemento imprescindible para su envejecimiento activo.

La población mundial se encuentra envejeciendo y el grupo de 65 años a más es el que crece más rápidamente, para el año 2050 una de cada seis personas en el mundo tendrá 65 a más años en comparación con la estadística del 2019 que es una de cada 11 personas. Las proyecciones entre el 2019 y el 2050, es que la población adulta mayor se duplicará en regiones del África y de América Latina y el Caribe. (Naciones Unidas, 2019) Y solo en el año 2018 se sobrepasó por primera vez en la historia el número de personas mayores de 65 años a más, al de los niños menores de cinco años de edad. Mientras que se proyecta que el número de personas mayores a 80 años se triplicará de 143 millones en el 2019 a 426 millones en el 2050.

En Perú la definición de persona adulta mayor comienza por la delimitación de la edad mayor o igual a 60 años en adelante. Existe un marco legal que respalda los derechos a una calidad de vida digna de las personas adultas mayores en cuatro ejes: derechos humanos, género, intergeneracional e intercultural, con base en la Ley de la persona adulta mayor $\mathrm{N}^{\mathrm{o}} 30490$ promulgada el año 2016, que da presencia y valor a la promoción y protección de los derechos de las personas adultas mayores, y que se encuentra bajo la rectoría del Ministerio de la Mujer y Poblaciones Vulnerables.

En el contexto peruano, en el año 2000 la población adulta mayor, llegaba a máximos de hasta 89 años de expectativa de vida, según el (Census, s.f.) https://www.census.gov/. Sin embargo, en los próximos diez años hasta el 2030, la expectativa rosará los 100 años de edad según la misma fuente consultada. Según las estadísticas del (INEI, s.f.), en el censo nacional de población y vivienda del 2017, la población absoluta de 65 años a más se incrementó en más de medio millón de personas entre 2007 y el 2017, pasando de 1'764,687 a 2'459,071. Lo que representa un incremento del $6.4 \%$ al $8.4 \%$.

En la investigación 'Del uso de las tecnologías de la comunicación a las destrezas en competencia mediática en las personas mayores', (Cruz, 2016), un documento exploratorio sobre la actitud de los adultos mayores frente al uso de las tecnologías de la información y de la comunicación en España, se aprecia que a pesar de haber mayores condiciones para el acceso a la Internet, aún existen muchos adultos mayores ajenos a adoptar las TIC como una nueva forma de comunicarse y socializar, eso se puede apreciar en la siguiente pregunta de la investigación: 'cree que es buen momento aprender informática después de la jubilación', un 90\% de los consultados reveló que nunca han mostrado interés, son los no usuarios. Mientras que el porcentaje restante, conformado por los que ya tenían conocimientos previos y los que aprendieron después, tenían estudios secundarios o superiores, de género masculino y hacían otras actividades.

Un análisis planteado por (Olivera, 2014) en su artículo 'Las características del adulto mayor peruano y las políticas de protección social', refiere que el proceso de envejecimiento en el futuro tendrá consecuencias para afrontar el sostenimiento de esta 
población, lo que incidiría en incrementar mayores condiciones de informalidad laboral y restricciones para el acceso a la salud. Si bien, el presente trabajo se enfoca en el marco de las TIC, es oportuno preguntarse si la falta de una adecuada inclusión educativa digital abonaría a favor de un impacto positivo para la economía de esta población.

\section{Metodología}

A fin de desarrollar un análisis de los documentos normativos de las instituciones del sector público del Estado peruano, la presente investigación se centra en el análisis de contenido de éstos. El análisis de contenido es una técnica de investigación científica pertinente que ayuda a tener una comprensión eficaz de un contexto simbólico, verbal y comunicacional (Krippendorff, 1990), por el cual se pueden hacer inferencias acerca de una determinada cuestión.

Las variables de análisis han sido elegidas de acuerdo con las terminologías más utilizadas en el contexto de la alfabetización digital como son: 'inclusión digital', 'alfabetización digital', 'educación virtual', 'educación digital', 'personas adultas mayores', 'adulto mayor' y 'personas de la tercera edad'.

Para determinar las unidades de contexto y de muestreo respectivamente, se realizó una revisión preliminar de los documentos normativos de instituciones rectoras como: la Presidencia del Consejo de Ministros de Perú, la Defensoría del Pueblo, la Oficina Nacional de Gobierno Electrónico e Informática (ONGEI), los Ministerios de Salud, Trabajo, Justicia, Educación, Transportes y Comunicaciones, Desarrollo e Inclusión Social, así como el de la Mujer y Poblaciones Vulnerables. Todos en su conjunto, forman parte de las instituciones que conforman los estamentos del poder Ejecutivo, a excepción de la Defensoría del Pueblo que es un ente autónomo.

De todos los mencionados se consideró como unidades de contexto a las instituciones rectoras en temas directamente relacionados con la investigación, es decir la educación, la comunicación, la tecnología y los adultos mayores. Quedando así los ministerios de: Educación (MINEDU), de la Mujer y Poblaciones Vulnerables (MIMP), de Transportes y Comunicaciones (MTC) y la Presidencia del Consejo de Ministros (PCM) como ente rector del ámbito tecnológico. Los cuales vienen a ser las únicas instituciones que hasta la fecha cuentan con normativa relacionada y vinculante a las condiciones y calidad de vida del público adulto mayor en referencia a la inclusión digital.

En cuanto a las unidades de muestreo, se consideran toda la normativa hallada en cada ente rector como son las leyes y sus reglamentos, así como los planes estratégicos nacionales de cada unidad de contexto. Es importante enfatizar que no todas tienen la misma cadena normativa, es decir, Ley, Reglamento, Plan, Estrategia.

La siguiente tabla está estructurada con una columna de observaciones que acompaña a cada unidad de muestreo a fin de destacar algún aporte oportuno que sume directa o indirectamente al presente trabajo académico. 
Tabla 1. Marco Normativo Peruano sobre la formación de capacidades para las Tecnologías de la Información y la Comunicación en el Público Adulto Mayor.

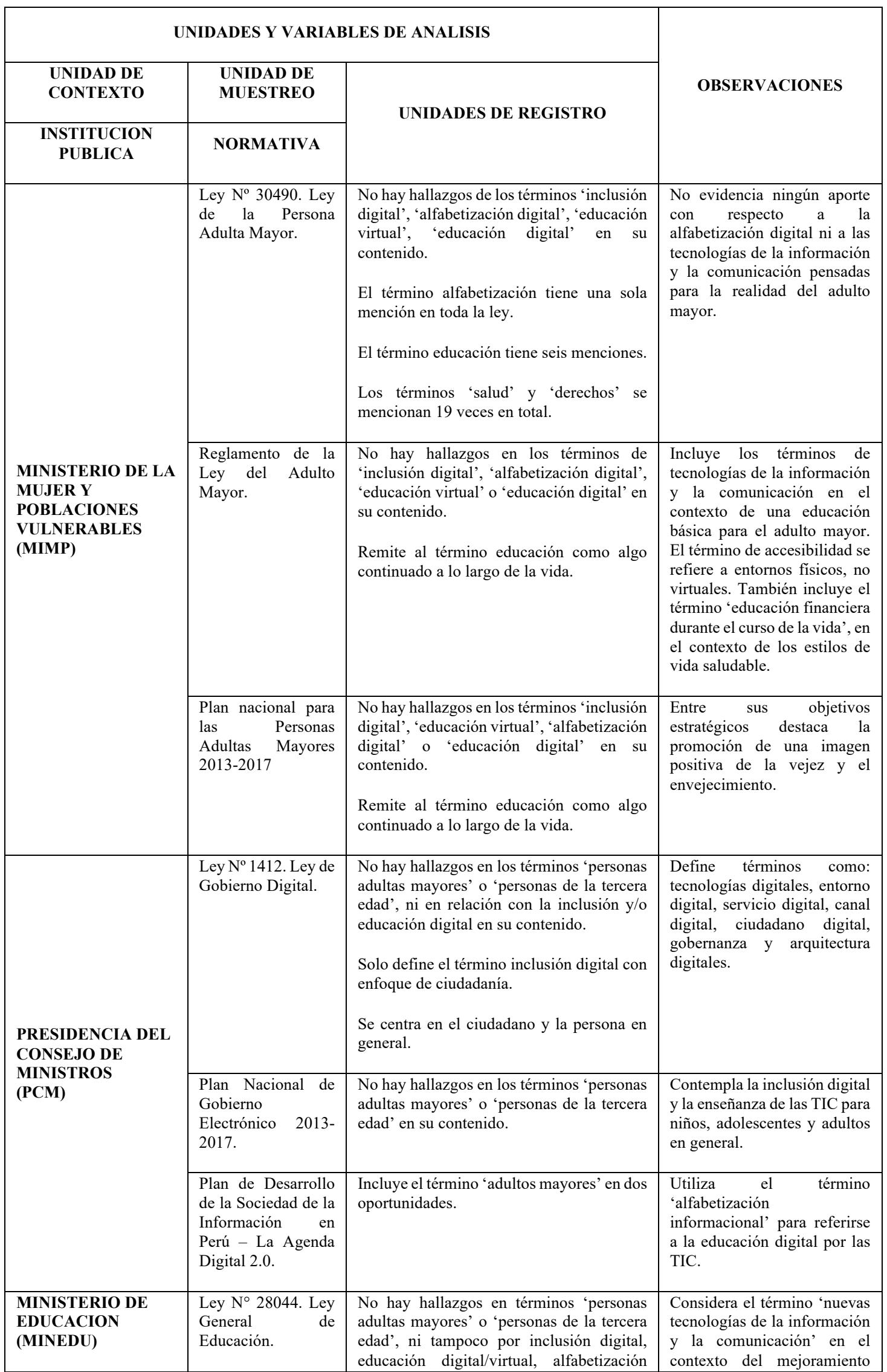




\begin{tabular}{|c|c|c|c|}
\hline & & $\begin{array}{l}\text { digital, competencia digital, entornos } \\
\text { virtuales, comunicación interactiva. }\end{array}$ & $\begin{array}{l}\text { del sistema educativo en } \\
\text { general. }\end{array}$ \\
\hline & $\begin{array}{l}\text { Estrategia Nacional } \\
\text { de las Tecnologías } \\
\text { Digitales en la } \\
\text { Educación Básica. }\end{array}$ & $\begin{array}{l}\text { No hay hallazgos en los términos 'personas } \\
\text { adultas mayores', 'personas de la tercera } \\
\text { edad' en su contenido. }\end{array}$ & $\begin{array}{l}\text { Destaca el modelo de } \\
\text { inteligencia digital para los } \\
\text { estudiantes y docentes. No } \\
\text { precisa rango de edades sólo } \\
\text { hasta } 25 \text { años a más. }\end{array}$ \\
\hline \multirow{3}{*}{$\begin{array}{l}\text { MINISTERIO DE } \\
\text { TRANSPORTES Y } \\
\text { COMUNICACIONES } \\
\text { (MTC) }\end{array}$} & $\begin{array}{l}\text { Ley } \mathrm{N}^{\circ} 29904 \text {. Ley } \\
\text { de Promoción de la } \\
\text { Banda Ancha. }\end{array}$ & $\begin{array}{l}\text { No hay hallazgos en los términos 'personas } \\
\text { adultas mayores' o 'personas de la tercera } \\
\text { edad', ni en relación con la inclusión y/o } \\
\text { educación digital en su contenido. }\end{array}$ & $\begin{array}{l}\text { El artículo } 23 \text { se refiere a la } \\
\text { alfabetización digital }\end{array}$ \\
\hline & $\begin{array}{l}\text { Reglamento de la } \\
\text { Ley de Promoción } \\
\text { de la Banda Ancha. }\end{array}$ & $\begin{array}{l}\text { Incluye el término 'adultos mayores' en el } \\
\text { artículo } \mathrm{N}^{\mathrm{o}} 44 .- \text { Alfabetización digital } \\
\text { referido al Título } \mathrm{V} \text { de la generación de } \\
\text { contenidos, aplicaciones y formación de } \\
\text { capacidades. }\end{array}$ & $\begin{array}{l}\text { Señala en el artículo } 6 \text { de los } \\
\text { principios para la política de } \\
\text { Banda Ancha, el principio de } \\
\text { accesibilidad para todas las } \\
\text { personas independientemente } \\
\text { de su edad entre otros. }\end{array}$ \\
\hline & $\begin{array}{l}\text { Plan Estratégico de } \\
\text { Gobierno Digital } \\
2020-22 \text { del MTC }\end{array}$ & $\begin{array}{l}\text { No hay hallazgos en los términos 'personas } \\
\text { adultas mayores' o 'personas de la tercera } \\
\text { edad', ni en relación con la inclusión y/o } \\
\text { educación, alfabetización digital en su } \\
\text { contenido. }\end{array}$ & $\begin{array}{l}\text { Promueve el proceso de la } \\
\text { transformación digital en } \\
\text { Perú. Promueve la inclusión } \\
\text { social entre los ciudadanos. } \\
\text { Su enfoque está basado en } \\
\text { proyectos del sector del } \\
\text { transporte y } \\
\text { comunicaciones. }\end{array}$ \\
\hline
\end{tabular}

Fuente: Elaboración propia

\section{Resultados}

Gracias al incremento de la esperanza de vida en el mundo, debido a los avances de la ciencia médica y la tecnología, el envejecimiento de la población mundial es una realidad del siglo XXI. Según la OMS, se calcula que para el 2050 la pirámide poblacional mundial de la tercera edad será superior a la de los jóvenes, en especial en países en desarrollo. En Perú, la realidad no es ajena, aunque se prevé que su población tenga un envejecimiento moderado, el país tiene muchos retos que afrontar para asegurar una esperanza de vida con calidad para la población adulta mayor, no solo en temas sanitarios, que es lo más importante, sino en temas como la educación continua y la inclusión.

En tal sentido, este trabajo busca dar un aporte hacia la alfabetización digital en el adulto mayor. Para lo cual se realizó un análisis de contenido del marco normativo de las instituciones ejecutoras del país, enfocado en los ejes de Educación, Tecnología y Comunicación y Poblaciones Vulnerables, cada uno de los cuales regidos por diferentes estamentos del Estado peruano.

En principio se comenzó con el análisis de la Ley de la Persona Adulta Mayor $N^{\circ}$ 30490 promulgada en el año 2016, la misma que señala al Ministerio de la Mujer y Poblaciones Vulnerables (MIDIS), como su ente rector para la promoción y protección de los derechos de las personas mayores. La ley se enfoca en 4 ejes: derechos humanos, genero, intergeneracional e intercultural. En su contenido, de tan solo 6 folios de extensión, se hace referencia al derecho a la promoción del acceso a programas y proyectos educativos con calidad a lo largo de la vida, el documento no precisaría ni referiría la importancia de la inclusión digital, ni el acceso al conocimiento de las TIC 
que podrían favorecer una adecuada alfabetización digital para este grupo etario. En cuanto al Reglamento de la Ley No 30490, Ley de la Persona Adulta Mayor, el análisis de contenido no arrojó ningún término relacionado a la relevancia de la alfabetización digital, la educación virtual o la inclusión digital en el adulto mayor, lo que explicaría la falta de mejores canales de la comunicación pública con la población adulta mayor. Otro documento de alcance nacional es el Plan Nacional para las Personas Adultas Mayores PLANPAM 2013 - 2017, el cual no hace referencia a ninguno de los términos inclusión digital, educación virtual, alfabetización o educación digitales en su contenido. Este documento marca los lineamientos de política nacional para la integración del Adulto Mayor tanto desde su participación e integración social como desde su inserción al mercado laboral, sin embargo, no haría ninguna referencia al acceso de las TIC para su mayor conocimiento e inclusión social.

Por su parte, la Presidencia del Consejo de Ministros - PCM-, tiene a su cargo la secretaría de Gobierno Digital cuyo enfoque es la atención en los trámites y servicios para los ciudadanos en general. Bajo su rectoría se encuentra la oficina de Gobierno Electrónico de Perú, que cuenta con dos herramientas normativas de gobierno, la Ley de Gobierno Digital N¹412 del año 2018, marco de gobernanza del gobierno digital en Perú y el Plan Nacional de Gobierno Electrónico 2013-2017. Ambos documentos no presentarían ninguna referencia directa a la población adulta mayor ni en relación con los términos de inclusión y/o educación digital en su contenido. La ley de Gobierno Digital define el término inclusión digital con enfoque de ciudadanía centrado en el ciudadano y las personas en general. Así mismo, se encuentra dentro de su rectoría el Plan de Desarrollo de la Sociedad de la Información en Perú - La Agenda Digital 2.0., el contenido de este documento sí hace referencia al menos una vez al término adultos mayores y al de alfabetización 'informacional' en lugar de alfabetización digital al referirse a la educación digital por las TIC en Perú. Salvo esta mención bastante sucinta y referencial, estos documentos estratégicos bajo la rectoría de la PCM dejarían como evidencia la falta de un enfoque inclusivo relevante donde se integre a este grupo etario que hoy en día es consumidor pasivo de las TIC.

El marco legal que respalda el acceso a la educación en Perú está regido por la Ley General de Educación $N^{\circ} 28044$, la cual señala en sus principios rectores la equidad e inclusión. En sus enunciados hace referencia a las nuevas Tecnologías de la Información y la Comunicación como un derecho de acceso a las personas en general incluyendo a las que tengan algún tipo de discapacidad. No obstante, la narrativa planteada en el documento no dejaría espacio para la inclusión digital pensada como tal para las personas adultas mayores. Lo mismo ocurre con el documento Estrategia Nacional de las Tecnologías Digitales en la educación básica, el cual no solo no hace referencia a este público etario, sino que utiliza los rangos de edades tan solo hasta 25 años a más.

Finalmente, el sector que contempla las regulaciones de las comunicaciones y el acceso a ellas como es el Ministerio de Transportes y Comunicaciones, rige tres documentos eje como son la Ley de Promoción de la Banda Ancha Ley N ${ }^{\circ} 29904$, el Reglamento de la Ley de Promoción de la Banda Ancha Ley $N^{\circ} 29904$ y el Plan 
Estratégico de Gobierno Digital 2020-22. En cuanto al Reglamento de la Ley N 29904, su contenido hace una referencia hacia los adultos mayores en el artículo $\mathrm{N}^{\mathrm{o}} 44$ Alfabetización Digital que debe ser desarrollado junto al Ministerio de Educación y la ONGEI para el diseño del Plan de Alfabetización Digital donde se incluya a este grupo vulnerable de la población.

\section{Conclusiones}

El presente estudio basado en el análisis de fuentes secundarias estructurales pone en evidencia que no existe un verdadero sentido de inclusión en la comunicación pública entre las organizaciones centrales y el público adulto mayor en Perú. Lo cual invisibiliza la necesaria importancia de incluir activamente a este grupo etario en la sociedad. Ello no favorecería en el presente como tampoco en el futuro al desarrollo de una calidad de vida digna para el envejecimiento activo de las personas adultas mayores en el marco del desarrollo de una Sociedad de la Información y el Conocimiento inclusiva en Perú. Se desprende que una de las consecuencias más evidentes que trae consigo el envejecimiento es la falta de una vida activa, la capacitación constante, la socialización permanente, por lo cual debieran ser los lineamientos rectores de gobierno los que abran espacios para un acceso libre a las tecnologías de la información y la comunicación para los adultos mayores indistintamente de su lugar de ubicación en el país.

En cuanto a las principales políticas de gobierno pensadas para una mejora en la calidad de vida de los peruanos, se puede concluir que las TIC no están siendo consideradas para integrar a las personas adultas mayores en su desarrollo continuo. En cuanto a los niveles de comunicación pública que se desprenden de todos los documentos estudiados, se observa que no hay un sentido claro para visibilizar la importancia de la alfabetización digital en el adulto mayor específicamente, ni de su inclusión como público activo y generador de sus propios contenidos, por lo tanto, tampoco habría una visibilidad real para enfatizar la importancia de las TIC en el desarrollo de estos.

El reto es integrar a este grupo etario como un nuevo público, hacerlo visible tanto como usuarios, así como generadores de sus propios contenidos, pues son personas capaces de construir sus propios significados digitales que sumen a la inclusión, la igualdad y la democracia del país. 


\section{Bibliografía}

Abad, L. (2017). Alfabetizacion mediatica para la e inclusion de los adultos mayores. Dykinson.

Aguado, J. (2004). e-comunicación. Dimensiones sociales y profesionales de la comunicación en los nuevos entornos tecnológicos. Sevilla: Comunicación Social Ediciones y Publicaciones.

Álvarez, J. (2013). Manejo de la comunicación organizacional: espacios, herramientas y tendencias en gestión de negocios. Madrid: Ediciones Díaz de Santos.

Area, M. (2015). Alfabetizacion Digital y la ciudadania del siglo XXI. 13.

Area, M. (s.f.). Alfabetización Digital y la ciudadanía del siglo XXI.

Castells, M. (2009). Comunicación y Poder. Madrid: Alianza Editorial.

Census, B. (s.f.). Census Bureau de Estados Unidos. Obtenido de https://www.census.gov/

Cruz, M. R. (2016). Del uso de las TIC's a las destrezas de los Adultos Mayores. Revista de Estudios para el Desarrollo Social de la Comunicación, 86. doi:doi:10.15213/redes.n12.p86

Fundación Telefónica. (2012). Alfabetización digital y competencias informacionales. Madrid: Ariel.

Gutierrez., M. (2003). Alfabetización Digital. Algo más que ratones y teclas. Barcelona: Gedisa.

INEI. (s.f.). Instituto Nacional de Estadística e Informática de Perú. Obtenido de https://www.inei.gob.pe/

Jenkins, H. (2008). Convergence Culture. La cultura de la convergencia de los medios de comunicación. Barcelona: Paidós.

Naciones Unidas, D. d. (17 de Julio de 2019). Press Release Perspectivas de la Poblacion Mundial 2019. Obtenido de https://population.un.org/wpp/Publications/Files/WPP2019_PressRelease_ES.pd f

Olivera, J. C. (2014). Las caracteristicas del adulto mayor peruano y las politicas de protección social. Economía, 38.

ONU. (2002). Informe de la Segunda Asamblea Mundial sobre el Envejecimiento. Madrid: Publicasion de las Naciones Unidas.

Paz, L. (2008). Alfabetización digital en el adulto maduro una estrategia para la inclusión social. Revista Unal, 16. 
PCM, P. d. (2011). Obtenido de https://sinia.minam.gob.pe: https://sinia.minam.gob.pe/documentos/plan-desarrollo-sociedad-informacionconocimiento-agenda-digital-20

Prensky, M. (2010). Nativos e Inmigrantes Digitales. Cuadernos SEK 2.0, 23.

Scolari, C. (2008). Hipermediaciones : Elementos para una teoría de la comunicación digital interactiva. Barcelona, España: Gedisa. Recuperado el abril de 2020, de ProQuest Ebook

Central, https://ebookcentral.proquest.com/lib/universidadcomplutenseebooks/detail.action?docID=4761457.

Travieso, J. P. (2008). la alfabetizacion digital como factor de inclusion social: una mirada critica. UOC Papers Revista sobre la sociedad del conocimiento(6), 9.

UIT, U. I. (2005). Cumbre Mundial sobre la Sociedad de la Información. Documentos Finales. Ginebra 2003 - Tunez 2005. Ginebra: Naciones Unidas.

\section{Nota}

${ }^{1}$ Luz María Flores Cabello es doctoranda en el programa de Periodismo de la Universidad Complutense de Madrid, con Maestría en Comunicación Social por la misma universidad. Es directora de la iniciativa periodística web www.terceraedaddigital.com enfocada en el público adulto mayor. Es investigadora del Instituto de Investigación de la facultad de Ciencias de Comunicación de la Universidad de San Martín de Porres. Sus líneas de investigación están referidas a la comunicación online, los canales sociales media y las asesorías de comunicación y relaciones públicas. 